\title{
Spectrally-Efficient Continuous Phase Modulations
}

\author{
Alan Barbieri, Dario Fertonani, Student Member, IEEE, and Giulio Colavolpe, Member, IEEE
}

\begin{abstract}
We investigate the spectral efficiency of continuous phase modulations (CPMs). To this end, we need an effective bandwidth definition for a CPM signal, whose power spectral density has in principle an infinite support. The definition we adopt is based on the spacing between adjacent carriers in a frequency division multiplexed CPM system. We consider the inter-channel interference, which depends on the channel spacing, and we evaluate the spectral efficiency achievable by a single-user receiver in the considered multi-channel scenario. We then optimize the channel spacing with the aim of maximizing the spectral efficiency, showing that impressive improvements with respect to the spectral efficiencies reported in the literature and obtained by heuristic approaches can be achieved.
\end{abstract}

Index Terms-Continuous phase modulation, interchannel interference, multiuser channels, information rate, spectral efficiency.

\section{INTRODUCTION}

C ONTINUOUS phase modulations (CPMs) form a class of constant envelope signaling, widely studied thanks to their appealing properties [1]. In particular, besides the robustness to non-linearities stemming from the constant envelope, some CPM formats are claimed to exhibit an excellent power and spectral efficiency [1], [2]. The spectral efficiency is an important quality figure for a modulation format, since it quantifies how many information bits per second can be loaded per unity of the available spectrum. To evaluate it, a suitable bandwidth definition is required. In fact, although the power of a CPM signal is typically concentrated in a small portion of the spectrum [3], the power spectral density (PSD) has rigorously an infinite support. The most commonly employed definition is that based on the power concentration, which defines the signal bandwidth as the bandwidth that contains a given fraction (this fraction being a parameter) of the overall power [4]-[6]. A different definition is used in [7], [8], which is based on the Carson's rule and exhibits a nice mathematical tractability. We also notice that some papers, as [9], investigated the information rate achievable by CPMs

Manuscript received May 20, 2008; revised August 18, 2008; accepted October 23, 2008. The associate editor coordinating the review of this paper and approving it for publication was D. Huang.

A. Barbieri and D. Fertonani are with Scuola Superiore Sant'Anna, Via G. Moruzzi 1, I-56124 Pisa (e-mail: a.barbieri@sssup.it; dario.fertonani@gmail.com).

G. Colavolpe is with Università di Parma, Dipartimento di Ingegneria dell'Informazione, Viale G. P. Usberti, 181A, I-43100 Parma, Italy (e-mail: giulio@unipr.it).

The paper has been presented in part at the IEEE International Symposium on Information Theory (ISIT'08), Tornto, Canada, July 2008, and at the 10th International Workshop on Signal Processing for Space Communications (SPSC'08), Rhodes Island, Greece, Oct. 2008.

This work is funded by the European Space Agency, ESA-ESTEC, Noordwijk, The Netherlands, under contract no. 19370/05/NL/JD.

Digital Object Identifier 10.1109/TWC.2009.080679 without accounting for the bandwidth and thus without giving any insight on the spectral efficiency.

A critical drawback of the above mentioned bandwidth definitions is that they are not prone to describe the impact of the power leakage outside the defined bandwidth in terms of interference caused to adjacent signals. In other words, the values of spectral efficiency they predict are nearly useless in a real multi-user scenario. As a matter of fact, from a communication system perspective, the final aim of any bandwidth definition is to evaluate the portion of the available spectrum taken up by a signal, so as to realize how far the adjacent channels should be placed from the signal under analysis when a frequency division multiplexed (FDM) system is considered. Based on this consideration, we consider an FDM-CPM transmission over a channel impaired by additive white Gaussian noise (AWGN), where all sub-channels are equally spaced and adopt the same CPM format, so that the effective bandwidth of a CPM signal is implicitly defined as the separation between two adjacent channels. Then, we evaluate the ultimate information rate achievable when singleuser detection is employed, that is, when each of the subchannels is processed by a dedicated receiver that neglects the inter-channel interference (ICI) - we will use the terms "channels" and "users" interchangeably. For the considered scenario, which turns out to be an instance of mismatched decoding [10], the information rate can be evaluated by means of the simulation-based algorithm described in [11], as a function of the spacing between the channels (i.e., the effective bandwidth). Hence, a method for evaluating the ultimate achievable spectral efficiency results, which improves the existing methods since it accounts for the presence of ICI.

By means of the proposed framework, we show that, for any given CPM format, the achievable spectral efficiency is significantly larger than that resulting from the conventional approaches, in which a fixed, non optimized spacing is considered. In particular, it turns out convenient to consider frequency spacings between the channels much lower than those deriving from the above mentioned bandwidth definitions, which can provide a better tradeoff between degradation of the information rate due to the ICI and usage of the available spectrum. Moreover, we show that the optimal spacing depends on the signal-to-noise ratio (SNR), that is like to say that the effective bandwidth of a CPM signal does depend on the SNR. Hence, for a given CPM format, the frequency spacing must be selected during design according to the operating SNR.

The framework proposed in this paper can be also used for selecting, based on information-theoretic arguments, a set of CPM formats suitable for a particular application, similarly to what has been done in [6] and [12] (see also [13]), where anyway the effect of ICI has been neglected-a comparison 
between such papers is fair since the same single-user receiver is considered. Moreover, we also investigate the impact of a multi-user receiver on the achievable spectral efficiency, in order to assess the performance improvements that can be obtained by employing receivers taking into account the interference due to the adjacent channels.

The remainder of this paper is organized as follows. In Section II we give the system model, while in Section III we address the related ultimate performance limits. In Section IV we describe a technique for optimizing the achievable spectral efficiency, whose effectiveness is proved by the simulation results reported in Section V. In this latter Section, other aspects are also investigated, namely, the possible performance improvement that could be obtained when either a correlated or non-uniformly distributed input sequence is fed to the CPM modulator or when a multi-user receiver is employed. Moreover, a convergence threshold analysis based on EXtrinsic Information Transfer (EXIT) charts [14] is carried out confirming the information theoretic results and demonstrating that the theoretical performance could be approached by rather standard coding schemes. Finally, Section VI gives some concluding remarks.

\section{System Model}

The complex envelope of a CPM signal has the form [1]

$$
s(t ; \mathbf{x})=\sqrt{\frac{2 E_{S}}{T}} \exp \left\{j 2 \pi h \sum_{n} x_{n} g(t-n T)\right\}
$$

in which $E_{S}$ is the energy per symbol, $T$ is the symbol interval, $h=r / p$ is the modulation index $(r$ and $p$ being relatively prime integers), the input symbols $\left\{x_{n}\right\}$, possibly correlated, take on values in the $M$-ary alphabet $\{ \pm 1, \pm 3, \ldots, \pm(M-1)\}$, and $\mathbf{x}=\left\{x_{n}\right\}$ denotes the input sequence. The function $g(t)$ is the phase-smoothing response and its derivative is the frequency pulse [1].

We consider a multi-channel FDM-CPM system, where all channels share the same CPM format and input alphabet. We assume that the input data streams are mutually independent, that all channels are received with the same power and are perfectly synchronized, i.e., no timing offsets are present between different channels (downlink assumption), and that the transmission channel only introduces AWGN, without any time or frequency selectivity. Although all these assumptions could be relaxed without affecting the main conclusions of this paper, for the application of the proposed method only the downlink assumption and that of absence of time or frequency selectivity can be removed. The overall complex low-pass equivalent received signal yields

$$
y(t)=\sum_{k=-K}^{K} s\left(t ; \mathbf{x}^{(k)}\right) e^{j 2 \pi k F t}+w(t)
$$

where $F>0$ represents the spacing (in $\mathrm{Hz}$ ) between the carriers, $w(t)$ is a zero-mean circularly-symmetric white Gaussian process with PSD $2 N_{0}$, and $2 K+1$ sub-channels are present.

We consider single-user detection, that is, each of the sub-channels is processed by a dedicated receiver that does not account for the ICI nor exchange any information with the receivers dedicated to the adjacent sub-channels. In the remaining of this paper we only consider single-user detection of the information stream $\mathbf{x}^{(0)}$. Let us rewrite (2) as

$$
y(t)=s\left(t ; \mathbf{x}^{(0)}\right)+w(t)+\sum_{k \neq 0} s\left(t ; \mathbf{x}^{(k)}\right) e^{j 2 \pi k F t}
$$

where the two different impairments experienced by the receiver, namely the background noise and the interference due to adjacent channels, are pointed out. Generally, for singleuser detection, it is assumed that the frequency spacing $F$ is large enough, such that the last term in (3) can be neglected in the bandwidth of interest. Here, we consider a more general approach, which consists of modelling the interference as a zero-mean circularly-symmetric white Gaussian process with PSD $2 N_{I}$, independent on the additive thermal noise-we point out that this approximation is exploited only by the receiver and is a design parameter, while in the actual channel the interference is of course generated as in (3). Hence, the channel model assumed by the receiver is

$$
y(t)=s\left(t ; \mathbf{x}^{(0)}\right)+n(t)
$$

where $n(t)$ is a zero-mean circularly-symmetric white Gaussian process with PSD $2\left(N_{0}+N_{I}\right)$. As explained later, since no obvious way for choosing a value of $N_{I}$ from the system parameters exists, this choice should be based on computer simulations. We will show that, by means of a suitable tuning of the parameter $N_{I}$, the auxiliary channel (4) can describe with better and better approximation the real multiuser channel (3).

\section{Ultimate Performance Limits}

We are interested in evaluating the ultimate performance limits achievable by a single-user receiver designed for the auxiliary channel (4) when the actual channel is that in (3), in terms of information rate and spectral efficiency. This issue, which is an instance of mismatched decoding [10], cannot be addressed in closed form, but can be solved by means of the simulation-based method described in [11], which only requires the existence of an algorithm for exact maximum a posteriori (MAP) symbol detection over the auxiliary channel. In the considered case, the auxiliary channel (4) is an AWGN channel, for which algorithms for MAP symbol detection can be derived with a frontend based on the Rimoldi decomposition [2] and the BCJR algorithm [15]. We denote by $\mathbf{y}^{(0)}$ any sequence of samples that are sufficient statistics for the detection of $\mathbf{x}^{(0)}$, for example the samples at the output of the bank of filters of the Rimoldi-based frontend.

The simulation-based method described in [11] allows to evaluate the achievable information rate (AIR) for the mismatched receiver, i.e.,

$I\left(\mathbf{x}^{(0)} ; \mathbf{y}^{(0)}\right)=\lim _{N \rightarrow \infty} \frac{1}{N} E\left\{\log \frac{p\left(\mathbf{y}^{(0) N} \mid \mathbf{x}^{(0) N}\right)}{p\left(\mathbf{y}^{(0) N}\right)}\right\}\left[\frac{\mathrm{b}}{\text { ch. use }}\right]$

where we use the superscript $N$ to remark that a sequence is truncated to its first $N$ elements. In (5), $p\left(\mathbf{y}^{(0) N} \mid \mathbf{x}^{(0) N}\right)$ and $p\left(\mathbf{y}^{(0) N}\right)$ are probability density functions according to the auxiliary channel model (4), while the outer statistical average is with respect to the input and output sequences evaluated according to the actual channel model (3) [11]. Both 


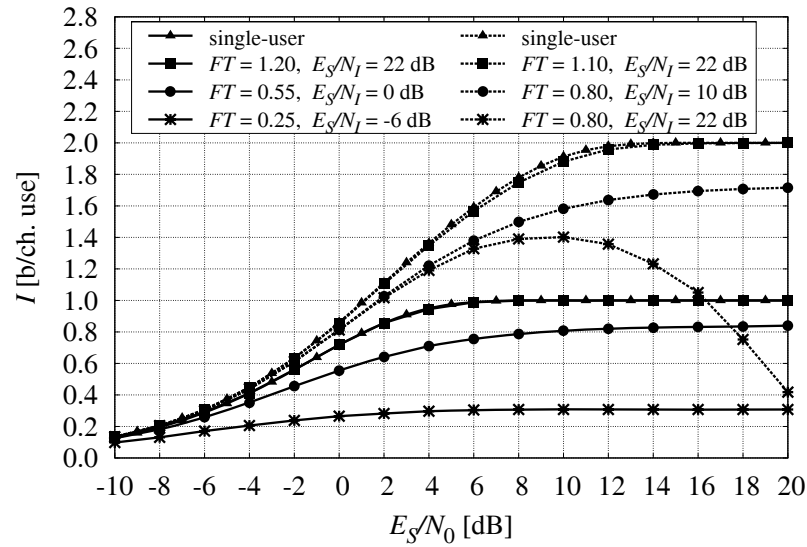

Fig. 1. Achievable information rate for an MSK modulation (solid curves) and a quaternary 2-RC modulation with $h=1 / 5$ (dashed curves).

$p\left(\mathbf{y}^{(0) N} \mid \mathbf{x}^{(0) N}\right)$ and $p\left(\mathbf{y}^{(0) N}\right)$ can be evaluated recursively through the forward recursion of the MAP detection algorithm matched to the auxiliary channel model [11]. Let us recall that the mismatched receiver can assure error-free transmissions when the transmission rate at the CPM modulator input does not exceed $I\left(\mathbf{x}^{(0)} ; \mathbf{y}^{(0)}\right)$ b/ch. use.

From a system viewpoint, the spectral efficiency, that is the amount of information transmitted per unity of time and per unity of bandwidth, is a more significant quality figure than the information rate. Hence, the derivation of the spectral efficiency for the considered system is given in the following. First, we notice that the overall information rate (in bits per channel use) achievable by the $2 K+1$ single-user receivers over the multi-user channel (2) is given by

$$
\sum_{k=-K}^{K} I\left(\mathbf{x}^{(k)} ; \mathbf{y}^{(k)}\right)
$$

where $\mathbf{y}^{(k)}$ is any sufficient statistic for the detection of $\mathbf{x}^{(k)}$, while the PSD of the multi-user signal is

$$
\sum_{k=-K}^{K} S_{X}(f-k F)
$$

$S_{X}(f)$ being the PSD of a single user. For increasingly large values of $K$ the boundary effects become negligible for both the overall information rate and the overall bandwidth. Hence, under the assumption of a large number of users, the achievable spectral efficiency (ASE) yields

$$
\eta=\frac{1}{F T} I\left(\mathbf{x}^{(0)} ; \mathbf{y}^{(0)}\right) \quad\left[\frac{\mathrm{b}}{\mathrm{s} \cdot \mathrm{Hz}}\right] .
$$

Hence, thanks to the assumption of a large number of users, we can keep focusing on the information stream $\mathbf{x}^{(0)}$ even for evaluating the ASE.

Some simulation results are reported in Fig. 1, which shows how the AIR varies with the value of $E_{S} / N_{0}$ when different values of the frequency spacing $F$ and the parameter $N_{I}$ are considered. As a benchmark, we added the corresponding single-user curves (equivalent to the mismatched case with infinite $F$ and $N_{I}=0$ ). These results refer to a binary

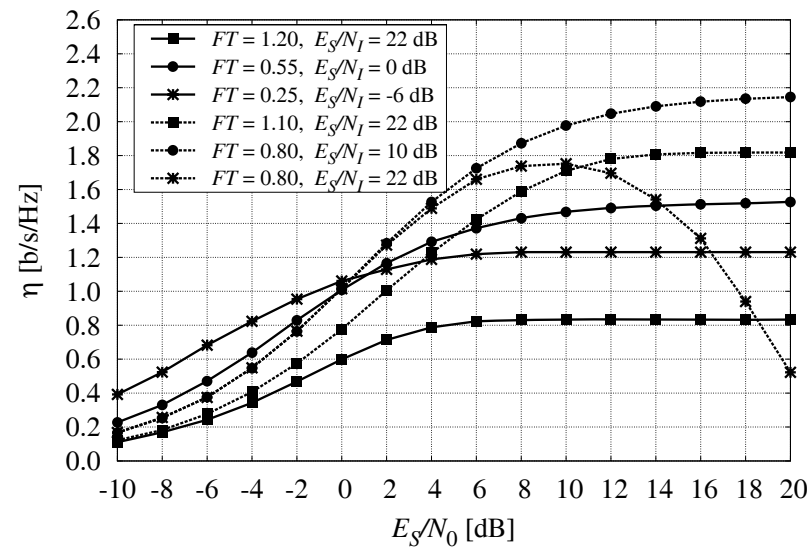

Fig. 2. Achievable spectral efficiency for an MSK modulation (solid curves) and a quaternary 2-RC modulation with $h=1 / 5$ (dashed curves).

1-REC modulation with $h=1 / 2$, often referred to as minimum-shift keying (MSK) modulation, and a quaternary 2-RC modulation with $h=1 / 5-L$-REC and $L$-RC denote respectively rectangular and raised cosine frequency pulses of length $L T$ [1]. Fig. 1 gives a quantitative evidence of the fact that the lower the value of $F$, the larger the interference due to the adjacent channels, and therefore the smaller the resulting AIR. In particular, when the value of $F$ is such that about the $99 \%$ of the signal power is concentrated in the bandwidth $[-F / 2, F / 2]$ [4], [6], [16], namely when $F T=1.2$ for the MSK modulation and $F T=1.1$ for the quaternary 2-RC modulation with $h=1 / 5$, the impact of the interference is nearly null. Also, Fig. 1 clarifies the impact of the parameter $N_{I}$, showing that, when it is significantly underestimated, the mismatch between the auxiliary channel and the actual one is such large that the AIR can decrease as the SNR increases. We remark again that the values of information rate shown in Fig. 1 are achievable by a MAP single-user receiver designed for the channel model (4).

On the other hand, very interesting insights are given by the results reported in Fig. 2, which shows the ASE corresponding to the same multi-user systems as in Fig. 1. These results clarify that the values of $F$ providing the best AIR are not those providing the best ASE, and thus that a careful design strategy, when the spectral efficiency is the key quality figure, should trade an intentional degradation in AIR for a larger ASE. For instance, in the case of the MSK modulation, the choice $F T=1.2$, based on the $99 \%$ of the signal power, provides a spectral efficiency dramatically lower than the choice $F T=0.55$. This fact confutes various previous works as [6], [7], [16], where the search for spectrally efficient schemes was carried out without exploiting the above mentioned tradeoff between AIR and ASE. An optimization algorithm able to exploit it is presented in the next section.

\section{Optimization of the Spectral EfFiciency}

Our aim is to find, for a given CPM format, the frequency spacing $F$ that provides the largest ASE. As clear from the results reported in Fig. 2, we should expect that the optimal spacing does depend on the SNR. Fig. 3, which refers to the 


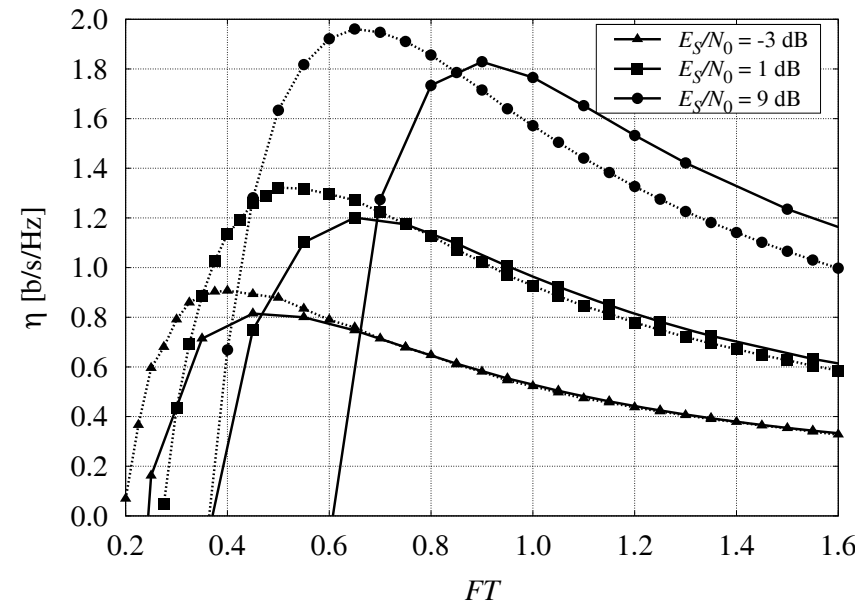

Fig. 3. Achievable spectral efficiency as a function of the spacing $F$, for a quaternary 2-RC modulation with $h=1 / 5$, when $N_{I}=0$ (solid curves) or $E_{S} / N_{I}=4 \mathrm{~dB}$ (dashed curves).

same quaternary modulation as in Fig. 1 and Fig. 2, gives more insights on this fact, suggesting that, as the signal-tonoise ratio increases, not only the ASE increases, but also the optimal spacing increases as well. The fact that at low values of the SNR the spacing can be "safely" reduced can be explained by realizing that an increase of the interfering power does not significantly affect the AIR as long as it is lower than the background noise power. The results reported in Fig. 3 were obtained either by setting $N_{I}=0$ (i.e., the impact of the interference is completely ignored by the receiver) or $E_{S} / N_{I}=E_{S} / \hat{N}_{I}=4 \mathrm{~dB}$, where $\hat{N}_{I}$ is the asymptotically optimal value of $N_{I}$, defined later. We remark that, especially at large values of $E_{S} / N_{0}$, the impact of $N_{I}$ on the optimal ASE is significant, thus a suitable optimization of $N_{I}$ is required. On the other hand, the importance of a fine optimization of $N_{I}$ decreases for increasingly large thermal noise power. For example, for very small values of the signalto-noise ratio, completely different values of $N_{I}$ lead to almost the same values of the optimal ASE.

For given $N_{I}$ and $E_{S} / N_{0}$, the properties of the function $\eta(F)$ cannot be easily studied in closed form, ${ }^{1}$ but it reads clear, by physical arguments, that it is bounded, continuous in $F$, and goes to zero when $F \rightarrow 0$ or $F \rightarrow \infty$. Hence, the function $\eta(F)$ has a maximum value-according to Fig. 3, one could also conjecture that there are no local maxima other than the global maximum. On the contrary, in the following we will see that, for some CPM formats (e.g., MSK), the function $\eta(F)$ exhibits two local maxima. Formally, for a given CPM format and a given value of $E_{S} / N_{0}$, the optimization problem consists of finding the maximum ASE

$$
\begin{aligned}
\eta_{\mathrm{M}}\left(E_{S} / N_{0}\right) & =\max _{F>0, N_{I} \geq 0} \eta\left(F, N_{I}, E_{S} / N_{0}\right) \\
& =\max _{F>0, N_{I} \geq 0} \frac{I\left(F, N_{I}, E_{S} / N_{0}\right)}{F T}
\end{aligned}
$$

which can be solved by evaluating $\eta\left(F, N_{I}, E_{S} / N_{0}\right)$ on a grid of values of $F$ and $N_{I}$ (coarse search), followed by an

\footnotetext{
${ }^{1}$ Although the functions AIR $I(\cdot)$ and ASE $\eta(\cdot)$ depend on various system parameters, in the following we will only explicitly indicate, from time to time, the parameters of interest for the relevant discussion.
}

interpolation of the obtained values (fine search). However, in order to speed up the simulation process, we decided to choose a single value $\hat{N}_{I}$ of $N_{I}$ for each CPM format, by means of the asymptotic (in the signal-to-noise ratio) joint optimization

$$
\hat{N}_{I}=\underset{N_{I}}{\operatorname{argmax}} \max _{F>0} \lim _{E_{S} / N_{0} \rightarrow \infty} \eta\left(F, N_{I}, E_{S} / N_{0}\right) .
$$

In other words, we use the following approximation

$$
\begin{aligned}
\eta_{\mathrm{M}}\left(E_{S} / N_{0}\right) & =\max _{F>0, N_{I} \geq 0} \eta\left(F, N_{I}, E_{S} / N_{0}\right) \\
& \simeq \max _{F>0} \eta\left(F, \hat{N}_{I}, E_{S} / N_{0}\right)
\end{aligned}
$$

which is very tight in all considered cases. We remark that the optimized spectral efficiencies shown in the next section can be (slightly) improved by jointly optimizing $F$ and $N_{I}$ for each value of $E_{S} / N_{0}$, as in (7).

So far, we addressed the optimization problem for a given value of $E_{S} / N_{0}$. On the other hand, a measure of the SNR more significant than $E_{S} / N_{0}$ is given by $E_{b} / N_{0}$, being $E_{b}$ the mean energy per information bit, for which the following equation holds

$$
E_{S}=I\left(E_{S}\right) E_{b}
$$

In order to solve the optimization problem for a given value of $E_{b} / N_{0}$, we employed the following technique. Firstly, the AIR is evaluated for some values of $F$ and $E_{S} / N_{0}$, denoted as $F_{1}<\ldots<F_{N}$ and $\left(E_{S} / N_{0}\right)_{1}<\ldots<\left(E_{S} / N_{0}\right)_{J}$, respectively. The two sets, including their cardinalities $N$ and $J$, must be designed so as to ensure an accurate enough sampling of the AIR, when the latter is interpreted as a function of $F$ and $E_{S} / N_{0} \cdot{ }^{2}$ For each $n=1, \ldots, N$, cubic spline interpolation can be used to obtain a continuous function of $E_{S} / N_{0}$, denoted as $I\left(F_{n} ; E_{S} / N_{0}\right)$. Then, given a value $\overline{E_{b} / N_{0}}$ of $E_{b} / N_{0}$, the following fixed point problems are solved in $E_{S} / N_{0}$ for each $n$

$$
\frac{E_{S}}{N_{0}}=I\left(F_{n}, \frac{E_{S}}{N_{0}}\right) \frac{\overline{E_{b}}}{N_{0}} .
$$

and the AIRs corresponding to the solutions are denoted by $I\left(F_{n}, \overline{E_{b} / N_{0}}\right)$. (Clearly, since there exists a lower bound on the achievable value for $E_{b} / N_{0}$, the above problem has no solution when unachievable values are considered.) Finally, a closed-form function $I\left(F, \overline{E_{b} / N_{0}}\right)$, with $F \in\left[F_{1} ; F_{N}\right]$, is obtained by interpolating the samples $I\left(F_{n}, \overline{E_{b} / N_{0}}\right)$ obtained above, and this function allows to easily solve the maximization problem.

\section{Numerical Results}

Some outcomes of computer simulations implementing the proposed optimization algorithm are reported and discussed in the following. In all of them, the optimization is carried out with respect to $E_{b} / N_{0}$.

Fig. 4 shows the maximum ASE $\eta_{M}$ as a function of $E_{b} / N_{0}$, for a set of CPM formats whose details are reported in the key. Similar comparisons can be used to select one or more

${ }^{2}$ In our numerical simulations we considered a rectangular sampling grid, i.e., $F_{n}=F_{1}+(n-1) \frac{F_{N}-F_{1}}{N-1}$, and similarly for $E_{S} / N_{0}$. The values of $N$ and $J$ were chosen through successive approximations: we select two initial values, we evaluate the AIR on the corresponding grid and, if the sampling rate turns out to be not sufficient, we double both $N$ and $J$, until a suitable sampling grid is found. 


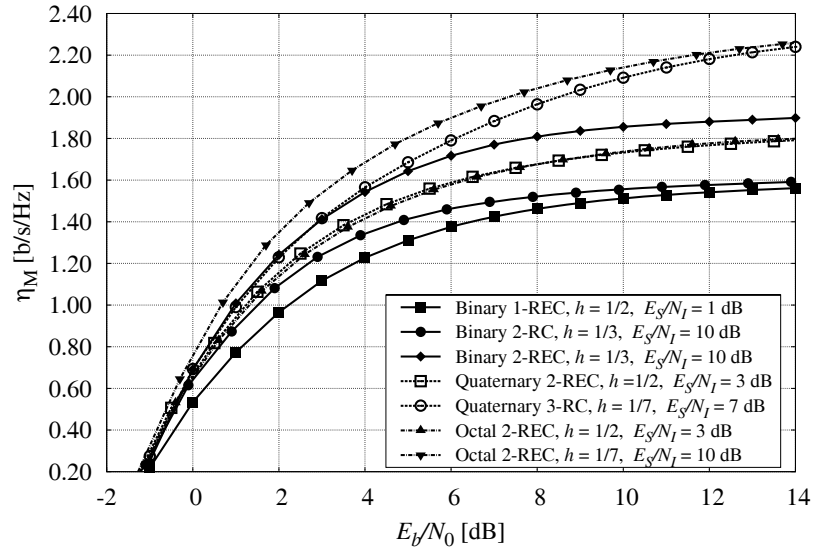

Fig. 4. Maximal achievable spectral efficiency as a function of $E_{b} / N_{0}$, for a set of binary, quaternary, and octal CPM schemes.

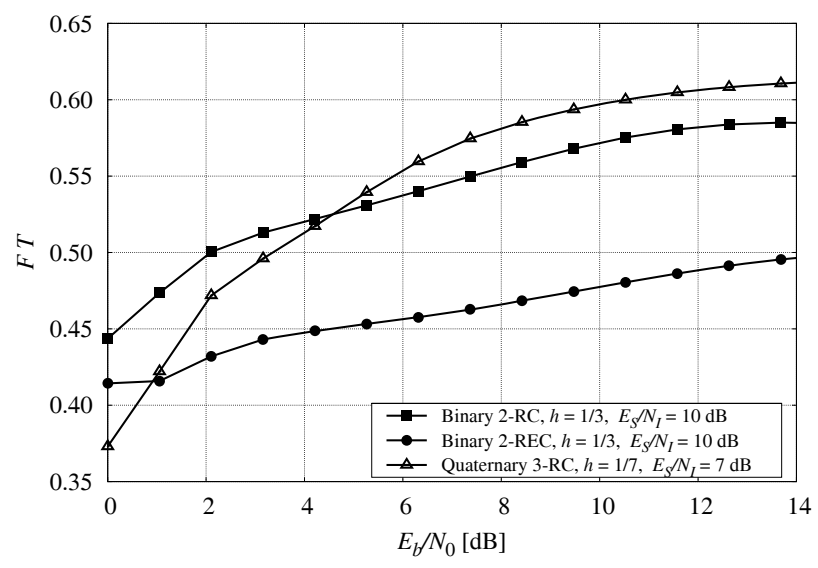

Fig. 5. Optimized normalized spacing as a function of $E_{b} / N_{0}$, for a selected set of CPM formats.

CPM formats out of a given set, according to an information theoretic performance metric (namely, the maximum spectral efficiency achievable by single-user detection). An interesting conclusion, confirmed by other simulation results not reported here, is that, once all the other parameters are set, modulations based on a REC frequency pulse always outperform the corresponding modulations based on a RC frequency pulse. This fact was unexpected, since it is widely believed that the RC frequency pulses provide better spectral properties [1], [6], [7], [12], [13], and is discussed later. In Fig. 5 the optimized normalized spacing $F T$ is shown for a subset of the CPM formats investigated in Fig. 4. This figure confirms a behavior conjectured in Section IV, namely the increase of the optimized spacing with the signal-to-noise ratio. On the other hand, although increasing with the SNR, the optimal spacings are much lower than the values related to the $99 \%$ of the signal power, again proving that a tradeoff between AIR and ASE is required. In fact, the normalized $99 \%$ bandwidth is 0.91 for the binary 2-RC $h=1 / 3$ format and 0.67 for the 2-REC $h=1 / 3$ format, thus much larger than the optimal spacing values shown in Fig 5. Moreover, we notice that the optimal spacing of the REC-based modulation is much smaller than that of the RC-based one. This explains why

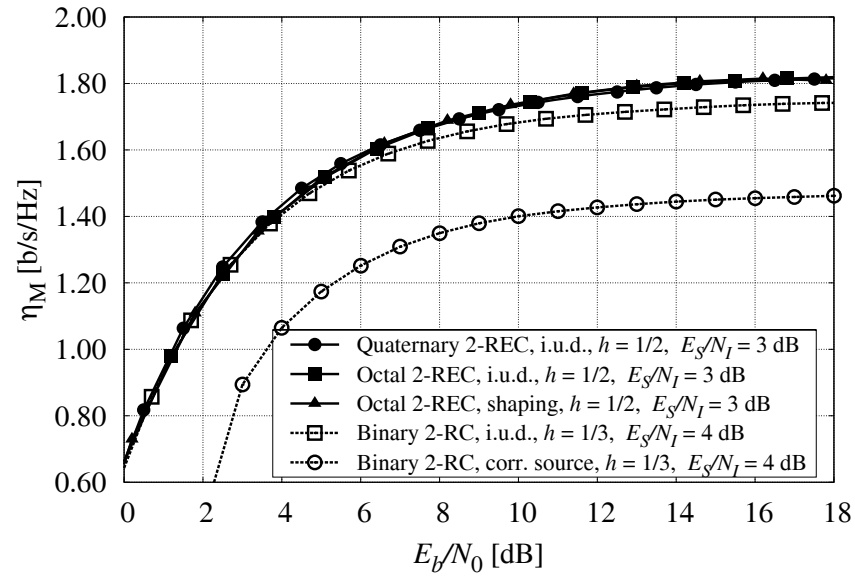

Fig. 6. Maximal achievable spectral efficiency as a function of $E_{b} / N_{0}$, for different CPM formats and type of inputs.

REC-based formats achieve higher spectral efficiencies than their RC-based counterpart. In fact, despite the spectrum of RC-based schemes looks "more compact", their robustness to ICI is lower than that of REC-based CPM formats-the robustness to intentional ICI was indeed not accounted for in the classical literature [1] nor in recent works as [6]-[8], [12], [13], [16] and for this reason RC-based schemes were considered more efficient.

Finally, we also analyzed a Gaussian Minimum Shift Keying (GMSK) modulation, characterized by $B T=0.3$ [17] as in the GSM cellular system, in the considered multiuser scenario. Although the relevant results are not reported here due to the lack of space, we obtained that the optimal normalized spacing, for asymptotically large values of the SNR, is about 0.55 , thus much smaller than the value 0.74 employed in GSM, corresponding to a gain in the ASE of about $25 \%$. For lower values of the SNR, the optimal spacing is even smaller. Therefore, a significant improvement of the ASE would have been achieved in GSM with a different choice of the spacing among the users in the frequency domain.

\section{A. Optimization of the Input Statistics}

In [8] it was shown that the asymptotic (i.e., for vanishing small noise power) spectral efficiency of a given CPM format can be considerably improved with a suitable shaping of the input sequence. Thanks to its mathematical tractability, the Carson's bandwidth was used to define the spectral efficiency. We considered one of the CPM formats studied in [8], namely an octal 2-REC with $h=1 / 2$, and we used the proposed method to evaluate the maximum ASE (through optimization of the spacing) when the optimized input distribution, proposed in [8], is used. For the above mentioned CPM format, in Fig. 6 we compare the maximum ASE for a quaternary and octal independent and uniformly distributed (i.u.d.) input, as well as for octal input optimized according to [8]. From the figure, it is clear that input optimization does not lead to any improvement in terms of maximum spectral efficiency, with respect to i.u.d. octal input. Even an i.u.d. quaternary input achieves the same performance. We notice that the asymptotic value of about $1.8 \mathrm{~b} / \mathrm{s} / \mathrm{Hz}$ obtained with the three different input formats, is obtained with completely different values of 
the spacing, namely $F T=0.96$ for the octal schemes and $F T=0.87$ for the quaternary scheme with i.u.d. inputs. In conclusion, when a suitable tradeoff between AIR and ASE is exploited, the input optimization proposed in [8] does not lead to any performance improvement.

The authors of [16] recently proposed to improve the spectral efficiency of a CPM system by introducing a suitable correlation among the symbols at the CPM modulator input. In particular, they analyzed 1st and 2nd order Markov sources, and optimized the transition probabilities so as to maximize the spectral efficiency, by employing the Carson's definition of the CPM bandwidth and neglecting the interference due to the adjacent channels in the frequency domain. ${ }^{3}$ One of the outcomes of that paper was that a suitable correlation of the input can dramatically reduce the occupied bandwidth, with only a negligible reduction of the information rate, and therefore that the overall spectral efficiency can be substantially improved. Thanks to the tools developed in this paper, we can verify in a more practical scenario the conclusions drawn in [16].

In Fig. 6, a binary 2-RC with $h=1 / 3$ modulation and an FDM multi-channel scenario are considered, with a singleuser receiver with $E_{S} / N_{I}=4 \mathrm{~dB}$. We compare the ASE as a function of $E_{b} / N_{0}$, for i.u.d. input and 1st order Markov correlated input, characterized by a transition probability $p_{T}=$ 0.21 (optimized according to the method of [16] at $E_{b} / N_{0}=4$ dB). Namely, we are increasing the probability of transmitting two consecutive equal symbols. Note that the correlated input, optimized according to [16], exhibits a largely reduced ASE with respect to the i.u.d. case, even at $E_{b} / N_{0}=4 \mathrm{~dB}$ (the SNR at which the transition probability has been optimized). Moreover, we verified that, when the interference due to the adjacent channels is considered, any value of the transition probability different from $p_{T}=1 / 2$ leads to a worse ASE. These and other simulation results, not shown here due to the lack of space, confirmed that giving up the memoryless input assumption and artificially introducing a correlation in the input sequence, always degrades the ASE in the considered multi-channel scenario.

\section{B. Optimization of the Phase Pulse}

In this section, we analyze how the performance of the considered multi-channel FDM-CPM system is affected by the frequency (or phase) pulse shape. In order to carry out this comparison without increasing too much the simulation complexity, it is mandatory to parameterize in some way the frequency pulse with no more than one or two parameters, and analyzing the performance for different values of those parameters. In particular, since up to now the rectangular pulse shape always gave the best performance, we generalize this pulse by defining a short-rectangular (denoted as SREC) frequency pulse according to

$$
q(t)= \begin{cases}\frac{1}{2 \delta L T} & \text { if }(1-\delta) \frac{L T}{2}<t<(1+\delta) \frac{L T}{2} \\ 0 & \text { otherwise }\end{cases}
$$

\footnotetext{
${ }^{3}$ The method proposed in [16] extends that proposed in [18] to the maximization of the spectral efficiency (rather than the information rate) for CPMs, according to the Carson's bandwidth definition and without taking into account the interference due to the adjacent channels.
}

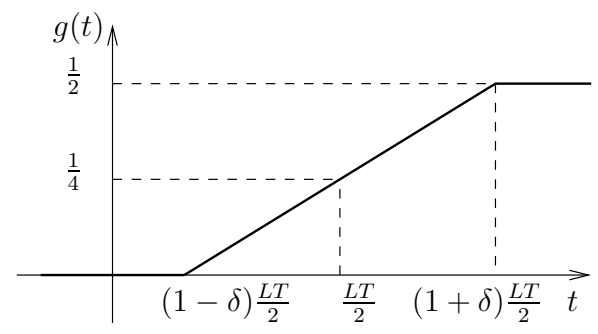

Fig. 7. SREC phase pulse, corresponding to the frequency pulse of eq. (11).

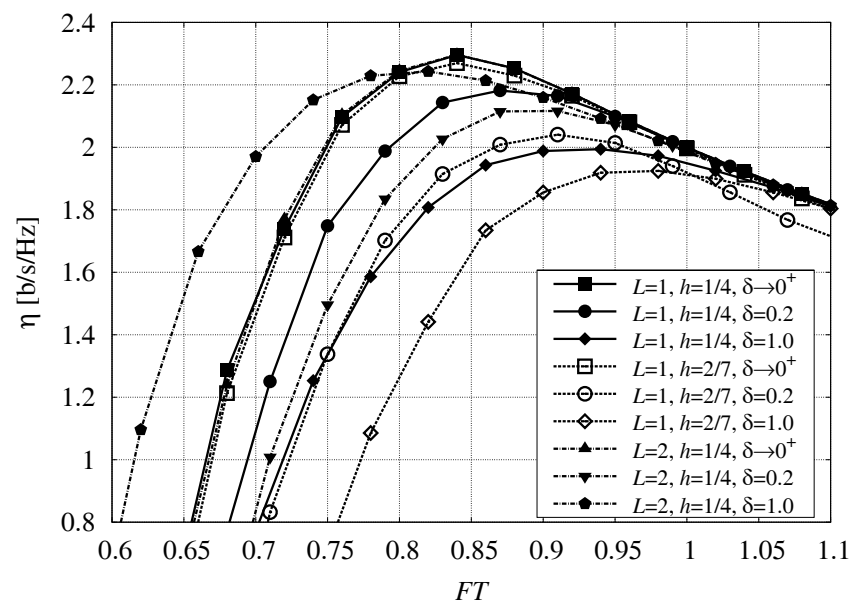

Fig. 8. Asymptotic ASE as a function of the normalized spacing FT and parameterized by $\delta$, for a quaternary modulation based on a SREC pulse, with different CPM parameters.

being $\delta \in(0,1]$ a parameter. The above pulse is rectangular of length $\delta L T$, and for $\delta=1$ the classical REC pulse is obtained. When $\delta \rightarrow 0^{+}$, the phase pulse becomes a step with an abrupt phase change at time $L T / 2$, and the phase continuity of the CPM modulation is lost. On the other hand, we point out that the second important property of CPMs, namely the constant envelope, is maintained for any $\delta>0$ when the SREC pulse is employed. To clarify this point, in Fig. 7 we show the phase pulse corresponding to the frequency pulse described by (11). It is worth noting that for $h=1 / M$ (being $M$ the input cardinality) and $\delta$ arbitrarily small, a differentially encoded $M$-ary phase shift keying ( $M$-PSK) with a rectangular pulse shape is in fact obtained. In general, for $h<\frac{1}{M-1}$, the $M$-ary information is univocally encoded as steep transitions between admissible values of the phase.

We now show an example of how the performance of the considered multi-channel FDM-CPM system is affected by the parameter $\delta$, when the frequency response defined in (11) is employed. As usual, we evaluated the spectral efficiency achievable by the considered single-user receiver. In Fig. 8 we show the asymptotic (i.e., for vanishing small additive noise) ASE as a function of the normalized spacing $F T$ and parameterized by $\delta$, for a quaternary modulation with $L$ equal to 1 or 2 and with two different values of $h$. A parameter $E_{S} / N_{I}=10 \mathrm{~dB}$ has been used in all the simulations. It is worth noting that the performance for $\delta=10^{-3}$, characterized by an abrupt phase change similar to that of a PSK modulation, always outperforms that for $\delta=1$ (namely, a classical REC CPM). This behavior is rather counter-intuitive according to 
the CPM literature, since it is widely believed that the phase continuity is mandatory in order to achieve very good spectral efficiencies. Although losing the phase continuity is expected to increase the bandwidth, and in fact the spectrum looks "less compact", the overall ASE is nevertheless improved, due to an increased robustness to adjacent channels interference.

We carried out simulations in many other scenarios (not shown here due to the lack of space), namely at low and medium SNRs, with different alphabet sizes and CPM parameters $L$ and $h$ (provided that $h$ is smaller than $\frac{1}{M-1}$ ), and with different values of $N_{I}$, and the vast majority of them confirmed the results discussed above. This looks to us a very interesting result, since it shows that, from an information-theoretic point of view, in the considered multi-channel scenario the phase continuity is not as beneficial as expected, and even shows that sometimes a substantial improvement of the ASE is achieved when an $M$-PSK modulation is used instead of a CPM. The existing CPM literature missed this point since, to the best of our knowledge, nobody has ever considered the effect of adjacent channels interference to the informationtheoretic performance of CPMs, as a function of the CPM format parameters and the spacing among the channels.

\section{EXIT Chart Analysis}

EXIT charts [14] are a powerful technique for the analysis and the design of iteratively decoded concatenations of component codes. They have been used successfully for the analysis and the design of serial and parallel concatenation of trellis codes [14], as well as for Low-Density Parity Check (LDPC) codes [19] and Repeat and Accumulate (RA) codes [20]. In order to confirm the results found in the previous sections and to show that the theoretical performance of the previous sections could be approached by rather standard coding schemes, we evaluated, by means of numerical simulations, the EXIT charts for a serially concatenated CPM scheme, where the inner component of the concatenation is the CPM modulator. All the curves reported in Fig. 9 have been obtained using a block length of 2048 code bits and, except for the curve marked "(5,7) CC" discussed below, at a signal-to-noise ratio $E_{S} / N_{0}=0.04 \mathrm{~dB}$. Two binary CPM formats with $L=2$ and REC or RC pulses, with $h=1 / 3$, were considered. In Fig. 9 the curves marked "no interf." refer to a single-user scenario, in which no interference due to adjacent channels is present. Note that in this case the EXIT curve of the RC-based format is strictly above that of the REC-based format, when all the other parameters $\left(L, M, h\right.$, and $\left.E_{S} / N_{0}\right)$ are fixed. As a consequence, in the interference-free case, irrespectively of the employed outer code the RC-based format would achieve an SNR convergence threshold possibly much smaller than its REC-based counterpart. This is well in line with the results found in the literature about serially concatenated, iteratively decoded CPM schemes.

On the other hand, the curves marked "interf." were instead obtained in the FDM multi-channel scenario considered in Section II, with a normalized spacing between adjacent channels $F T=0.43$. The single-user MAP receiver for the AWGN channel has been employed, with an increased assumed noise variance (namely, $E_{S} / N_{I}=10 \mathrm{~dB}$ ). Note that in this case

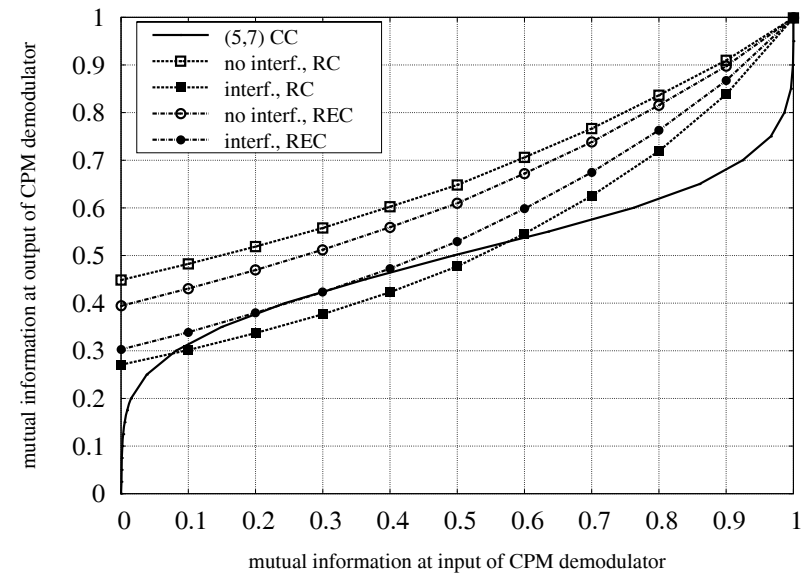

Fig. 9. EXIT charts of the considered serially concatenated CPM scheme, for a 2-REC and a 2-RC binary modulation with $h=1 / 3$, at $E_{S} / N_{0}=0.04$ dB.

the EXIT curves noticeably decrease, due to the impact of the adjacent interference, and therefore the convergence threshold would necessarily increase, irrespectively of the employed outer code. However, it is worth noting that the decrease of the REC-based EXIT curve is much less pronounced that that of the RC-based format, due to the larger robustness of the REC schemes to the interference coming from the adjacent channels in the frequency domain. This is a further confirmation of the results obtained so far through the proposed scheme for the evaluation of the achievable spectral efficiency.

Finally, in Fig. 9 we also added the EXIT curve of a rate$1 / 2$ convolutional code $(\mathrm{CC})$ with generators $(5,7)$, employed as outer code in the concatenated scheme. Note that, since the EXIT curve of the 2-REC with interference scenario is strictly (although slightly) above the EXIT curve of the outer code, according to the EXIT charts literature we are in the so-called "open tunnel" scenario. The value $E_{S} / N_{0}=0.04 \mathrm{~dB}$ was indeed chosen since it is the minimum value of the SNR that guarantees the open tunnel condition (i.e., convergence threshold). We remark that, according to our simulation results, a 2-REC binary modulation with $h=1 / 3$, at $E_{S} / N_{0}=-1.33$ $\mathrm{dB}$ achieves its maximum spectral efficiency using a spacing $F T=0.43$ and a corresponding AIR of $1 / 2$. Hence, the convergence threshold obtained through the EXIT analysis is about $1.37 \mathrm{~dB}$ worse than that predicted by the method proposed in Section IV. This loss is mainly due to the fact that the outer code has been chosen arbitrarily (i.e., the $(5,7) \mathrm{CC}$ ) and has not been optimized and not to the interference, since the very same behavior could be observed when comparing the ultimate performance limits and the effective convergence threshold predicted by the EXIT analysis for the interferencefree case. In Table I the values of $E_{b} / N_{0}$ for which an AIR of $1 / 2$ is achieved, and the corresponding convergence thresholds evaluated by means of the EXIT chart analysis when the considered CC is employed as outer code, are shown for the four scenarios considered in Fig. 9. We finally point out that, for the reasons discussed above, the EXIT charts can be successfully used for the design of the outer code with the aim of minimizing the convergence threshold, for any given inner CPM modulator and spacing between adjacent channels. 
TABLE I

CONVERGENCE THRESHOLD VERSUS INFORMATION THEORETIC ANALYSIS, FOR THE FOUR SCENARIOS CONSIDERED IN FIG. 9.

\begin{tabular}{|l|c|c|c|c|}
\hline \hline & $\begin{array}{c}\text { 2-REC } \\
\text { single-user }\end{array}$ & $\begin{array}{c}\text { 2-RC } \\
\text { single-user }\end{array}$ & $\begin{array}{c}\text { 2-REC } \\
\text { multi-user }\end{array}$ & $\begin{array}{c}2-\mathrm{RC} \\
\text { multi-user }\end{array}$ \\
\hline \hline Information-theoretic threshold & $-2.11 \mathrm{~dB}$ & $-2.52 \mathrm{~dB}$ & $-1.39 \mathrm{~dB}$ & $-0.81 \mathrm{~dB}$ \\
\hline Convergence threshold (EXIT chart) & $-1.07 \mathrm{~dB}$ & $-1.48 \mathrm{~dB}$ & $0.04 \mathrm{~dB}$ & $0.96 \mathrm{~dB}$ \\
\hline Gap & $1.04 \mathrm{~dB}$ & $1.04 \mathrm{~dB}$ & $1.43 \mathrm{~dB}$ & $1.77 \mathrm{~dB}$ \\
\hline \hline
\end{tabular}

\section{Multi-User Demodulator}

All the results presented so far rely upon the fundamental assumption about the channel model assumed by the receiver, described by eq. (4). Although the assumption of a single-user auxiliary channel has led to very useful results, it is instructive to investigate a more general auxiliary channel model, and thus a different receiver prototype. To this purpose, the channel model assumed by the receiver is now

$$
y(t)=\sum_{k=-J}^{J} s\left(t ; \mathbf{x}^{(k)}\right)+n(t)
$$

where the integer $J>0$ is a design parameter and $n(t)$ a circularly-symmetric AWGN process with PSD $2\left(N_{0}+N_{I}\right)$. In other words, we are here considering a multi-user receiver for the user with index 0 that takes into account the $J$ adjacent signals on each side as well-we again point out that this approximation is exploited only by the receiver, while in the actual channel the interference is generated as in (3). The exact MAP receiver for the multi-user channel (12) must be developed in order to find the ASE in the new scenario. The benefit of employing the multi-user auxiliary channel model when evaluating the ASE is two-fold: first, it allows to evaluate the performance degradation due to the use of the trivial single-user receiver, despite the presence of a strong adjacent channel interference, with respect to a more involved multi-user receiver, which is more "matched" to the real channel. Second, for increasingly large $J$ it gives a practical performance upper bound when low-complexity approximate multi-user receivers, for example based on linear equalization or interference cancellation, are employed.

In Fig. 10 the spectral efficiency achieved by a MAP receiver assuming the channel model (12) with $J=1$, when employed on the multi-user channel with infinite users, is shown as a function of the normalized spacing, for vanishing small additive noise power and two selected CPM formats. For comparison purposes, the corresponding spectral efficiencies achieved by the single-user receiver, employed on the same multi-user channel, are shown. It is worth noting that, as expected, the multi-user receiver achieves a substantially higher ASE than the corresponding single-user receiver, and that the optimal spacing decreases, at least for the considered 2-RC format. The latter effect stems from the increased robustness of the multi-user receiver (even when $J=1$ ) to the adjacent channel interference, which allows to improve the spectral efficiency through a remarkable reduction of the spacing.

Finally, we point out that when the MSK format is considered, irrespectively of the employed auxiliary channel model and corresponding receiver prototype, a marked local minima of the ASE appears for $F T=0.5$. This is due to the fact that

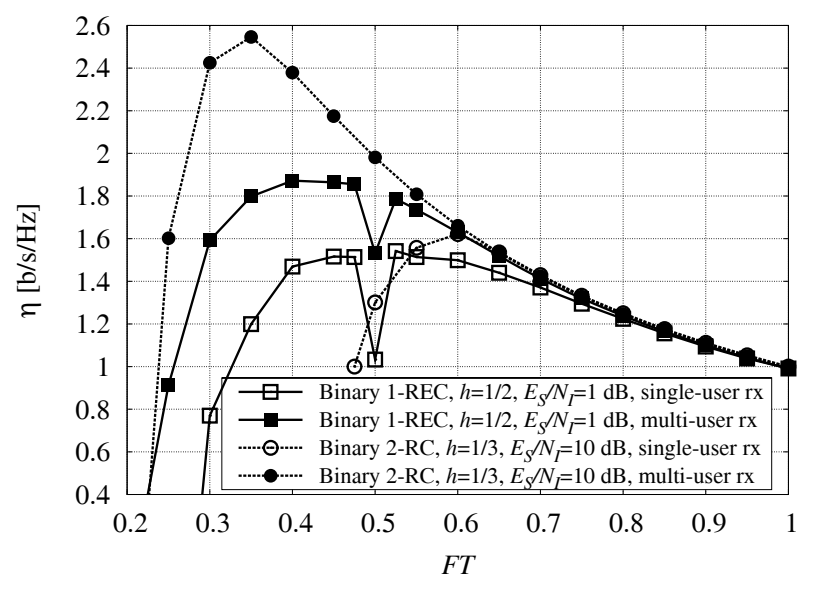

Fig. 10. Achievable spectral efficiency as a function of the spacing $F$, for single-user and multi-user receivers, when $N_{0}=0$.

two adjacent MSK channels, placed at a distance $F T=0.5$ in the frequency domain, strongly interfere one of each other since the waveform associated to the transmitted symbol +1 of one channel is indistinguishable from the waveform associated to the transmitted symbol -1 of the adjacent channel in the same symbol period. Note that this behavior depends on the downlink assumption (i.e., perfect timing synchronization of all the channels), thus the local minima of the spectral efficiency disappears when an independent random timing offset is applied to every channel.

\section{CONCLUSIONS}

We have considered an FDM-CPM multi-user system and we have evaluated the information rate achievable by a mismatched receiver, namely a single-user MAP detector employed in the considered multi-user scenario. By defining the bandwidth as the spacing between adjacent carriers, we have described a technique for selecting the system parameters providing the best spectral efficiency. Several insights have been given which confute well established beliefs found in the literature. In particular, we have shown that a suitable tradeoff between AIR and ASE is required, that the REC-based modulations outperform the RC-based ones, and that the input optimizations proposed in [8] and [16] are not effective in the considered multi-user scenario.

\section{REFERENCES}

[1] J. Anderson, T. Aulin, and C.-E. Sundberg, Digital Phase Modulation. New York: Plenum Press, 1986.

[2] B. E. Rimoldi, "A decomposition approach to CPM," IEEE Trans. Inform. Theory, vol. 34, pp. 260-270, Mar. 1988. 
[3] T. Aulin, N. Rydbeck, and C.-E. W. Sundberg, "Continuous phase modulation-part II: partial response signaling," IEEE Trans. Commun., vol. 29, pp. 210-225, Mar. 1981.

[4] P. Moqvist and T. M. Aulin, "Serially concatenated continuous phase modulation with iterative decoding," IEEE Trans. Commun., vol. 49, pp. 1901-1915, Nov. 2001.

[5] K. Padmanabham, S. Ranganathan, S. P. Sundaravaradhan, and O. M. Collins, "General CPM and its capacity," in Proc. IEEE International Symposium Inform. Theory, pp. 750-754, Sept. 2005.

[6] S. Benedetto, G. Montorsi, A. Perotti, and A. Tarable, "Optimization of CPM pragmatic capacity," in Proc. IEEE Global Telecommun. Conf., Nov. 2007.

[7] C. H. Kuo and K. M. Chugg, "On the bandwidth efficiency of CPM signals," in Proc. IEEE Military Comm. Conf. (MILCOM), pp. 218-224, Oct.-Nov. 2004.

[8] C. H. Kuo and K. M. Chugg, "Improving the bandwidth efficiency and of CPM signals via shaping and iterative detection," in Proc. IEEE Military Comm. Conf. (MILCOM), pp. 596-602, Oct. 2005.

[9] S. Benedetto, G. Montorsi, A. Perotti, and A. Tarable, "A pragmatic approach to coded continuous-phase modulation," in Proc. Inform. Theory Appl. Workshop, pp. 36-40, Jan.-Feb. 2007.

[10] N. Merhav, G. Kaplan, A. Lapidoth, and S. S. Shitz, "On information rates for mismatched decoders," IEEE Trans. Inform. Theory, vol. 40, pp. 1953-1967, Nov. 1994.

[11] D. M. Arnold, H.-A. Loeliger, P. O. Vontobel, A. Kavčic̀, and W. Zeng, "Simulation-based computation of information rates for channels with memory," IEEE Trans. Inform. Theory, vol. 52, pp. 3498-3508, Aug. 2006 .
[12] A. Graell i Amat, C. A. Nour, and C. Douillard, "Serially concatenated continuous phase modulation for satellite communications," submitted to IEEE Trans. Wireless Commun., July 2007.

[13] A. Graell i Amat, C. A. Nour, and C. Douillard, "Serially concatenated continuous phase modulation with extended BCH codes," in Proc. IEEE Information Theory Workshop Information Theory Wireless Net., pp. 15, July 2007.

[14] S. ten Brink, "Convergence behavior of iteratively decoded parallel concatenated codes," IEEE Trans. Commun., vol. 49, pp. 1727-1737, Oct. 2001.

[15] L. R. Bahl, J. Cocke, F. Jelinek, and J. Raviv, "Optimal decoding of linear codes for minimizing symbol error rate," IEEE Trans. Inform. Theory, vol. 20, pp. 284-287, Mar. 1974.

[16] A. Barbieri, A. Cero, A. Piemontese, and G. Colavolpe, "Markov capacity of continuous phase modulations," in Proc. IEEE International Symposium Inform. Theory, Nice, France, June 2007.

[17] K. Murota and K. Hirade, "GMSK modulation for digital mobile radio telephony," IEEE Trans. Commun., vol. 29, pp. 1044-1050, July 1981.

[18] A. Kavčic̀, X. Ma, and N. Varnica, "Matched information rate codes for partial response channels," IEEE Trans. Inform. Theory, vol. 51, pp. 973-989, Mar. 2005.

[19] S. ten Brink, G. Kramer, and A. Ashikhmin, "Design of low-density parity-check codes for modulation and detection," IEEE Trans. Commun., vol. 52, pp. 670-678, Apr. 2004.

[20] S. ten Brink and G. Kramer, "Design of repeat-accumulate codes for iterative detection and decoding," IEEE Trans. Signal Processing, vol. 51, pp. 2764-2772, Nov. 2003. 